\title{
Two classes of ethidium-bromide-resistant mutants of Streptomyces lividans 66
}

\author{
Li-Fong Lee, ${ }^{1}$ Yi-Jiun Huang ${ }^{2}$ and Carton W. Chen ${ }^{3}$ \\ Author for correspondence: Carton W. Chen. Tel: +8862826 7040. Fax: +88628264930 . \\ e-mail: cwchen@ym.edu.tw
}

Institute of Biochemistry, ${ }^{1}$ Institute of Microbiology \& Immunology, ${ }^{2}$ and Institute of Genetics, ${ }^{3}$ National Yang-Ming University, Taipei 112, Taiwan

\begin{abstract}
Five spontaneous mutants of Streptomyces lividans TK64 resistant to 5 or $15 \mu \mathrm{M}$ ethidium bromide (EB) were isolated, and the corresponding mutations were mapped to two different chromosomal locations. Both types of mutations conferred unselected resistance to several basic dyes and norfloxacin. The strain with the low-level resistance exhibited wild-type levels of EB uptake and energy-dependent efflux, and the resistance mechanism is unclear. The highly resistant mutants, which additionally were resistant to phosphonium ions, had a reduced accumulation and an increased efflux of EB, reminiscent of a mammalian multidrug resistance efflux pump.
\end{abstract}

Keywords: Streptomyces, ethidium bromide, multidrug resistance

\section{INTRODUCTION}

DNA-intercalating agents, such as ethidium bromide (EB), acriflavine and acridine orange, are basic compounds containing complex hydrophobic rings that can insert between the base pairs in the double helix. These DNA intercalators appear to enter the cells freely and interfere with a large number of processes, such as replication, transcription, translation, recombination (Seto \& Tomasz, 1977), RNA splicing (Tanner \& Cech, 1985), maintenance of plasmids, and formation of Z-DNA (Walker et al., 1985; Hardin et al., 1988). They also generate frameshift mutations (Brenner et al., 1961; Crick et al., 1961). In Gram-positive filamentous bacteria of the genus Streptomyces they also induce extensive DNA deletions and amplifications (Schrempf, 1983; Hintermann et al., 1984) at the terminal (unstable) regions of the linear chromosomes (Redenbach et al., 1993). Little is known about how these agents cause plasmid curing or rearrangement of chromosomal DNA. These compounds cause cell death, but it is not known whether the lethality is due to mutagenicity or to some other toxic effect.

Many bacteria, including Escherichia coli, are resistant to DNA-intercalating compounds. Sensitive mutants have been isolated with a defective energy-dependent efflux system. Genes that appear to be involved in the efflux systems have been cloned from the chromosome or plasmids of E. coli and other bacteria (reviewed by Lewis, 1994; Ma et al., 1994; Nikaido, 1994). The protein products encoded by these genes are cell membrane

Abbreviations: $E B$, ethidium bromide; $C C C P$, carbonyl cyanide $m$ chlorophenylhydrazone. transporters, and may be grouped into four families according to their supramolecular assembly, mechanism of action, and sequence homology (Nikaido, 1994). Each system confers resistance to remarkably broad (and often overlapping) spectra of agents, only some of which are structurally related. The most common targets for these pumps are basic dyes, hydrophobic antibiotics, quaternary ammonium compounds, fluoroquinolones and detergents.

In contrast, Gram-positive bacteria, such as Bacillus subtilis and Stapbylococcus aureus, are more sensitive to EB (Nikaido, 1994), and resistant mutants may be selected. Neyfakh et al. (1991) isolated Bacillus subtilis mutants (bmr) resistant to rhodamine- $6 \mathrm{G}$, which were cross-resistant to EB. The increased resistance appeared to be mediated by an elevated efflux. These authors cloned the resistance gene, and found it to encode a 389 amino acid protein with 12 putative transmembrane domains. The $\mathrm{Bmr}$ protein closely resembled the tetracycline-efflux (TetR) proteins encoded by $\operatorname{Tn} 10, \operatorname{Tn} 721$ and $\mathrm{pBR} 322$, in agreement with its putative role in drug efflux.

In Stapbylococcus aureus, determinants of resistance to quaternary ammonium compounds and basic dyes have been found on various plasmids. The resistance determinant, qac $A$, on plasmid pSK1 also mediates an efflux system (Tennent $e t$ al., 1985, 1989). gac $A$ (Sasatsu et al., 1989; Rouch et al., 1990) encodes a 514 amino acid protein, which also resembles TetR and Bmr. This type of drug resistance, mediated by efflux, is similar to the so called 'multidrug resistance' in cancer cells conferred by an elevated level of membrane-bound P-glycoprotein that pumps out cytotoxic drugs of various kinds, including DNA-intercalating compounds. 
Here we report that wild-type Streptomyces lividans tolerates only low concentrations of EB, and resistance is not induced by pre-exposure to a sublethal level of EB. Spontaneous mutants of $S$. lividans resistant to elevated $\mathrm{EB}$ concentrations were isolated and characterized. These mutants were grouped into two classes with respect to their efficiencies of EB efflux and substrate spectra, and the chromosomal locations of the mutations. One class of mutants exhibited increased efflux of EB, and the other did not. These results revealed at least two separate genetic systems involved in multidrug resistance.

\section{METHODS}

Bacterial strains and plasmids. The $S$. lividans strains are listed in Table 1. Streptomyces cultures were grown in $\mathrm{YM}(0.3 \%, \mathrm{w} / \mathrm{v}$, yeast extract, $0 \cdot 3 \%$, w/v, malt extract, $2 \%$, w/v, agar), $\mathrm{CM}$ (YM plus $0.5 \%$, w/v, peptone, $1 \%$, w/v, glucose and $5 \mathrm{mM}$ $\mathrm{MgCl}_{2}$; Dyson \& Schrempf, 1987), MM or R2 (Hopwood et al., 1985) solid medium, or TSB liquid medium (Hopwood $t$ t al., 1985). Required amino acid and antibiotic supplements were added to MM as described by Hopwood et al. (1985).

Chemicals. Lysozyme, agarose, streptomycin, spectinomycin, acridine orange, proflavine, acriflavine, methyl green, rhodamine- $6 \mathrm{G}$, benzalkonium chloride, toluidine blue $\mathrm{O}$, norfloxacin and carbonyl cyanide $m$-chlorophenylhydrazone (CCCP) were purchased from Sigma; cetyltrimethylammonium bromide and tetraphenylphosphonium bromide from Aldrich; methyl triphenylphosphonium bromide from Fluka; EB and methylene blue from E. Merck; pyronine $\mathrm{Y}$ from Allied Chemical; crystal violet from J. T. Baker; and restriction enzyme AseI from New England Biolabs.

Drug sensitivity test. For survival counts, S. lividans spore suspensions were plated on YM plates containing EB and incubated in the dark at $30^{\circ} \mathrm{C}$; surviving colonies were counted after $2-3$ d. Semi-quantitative tests were performed by placing $8 \mathrm{~mm}$ filter disks (Toyo Adventec, Toyo Roshi) containing $25 \mu \mathrm{l}$ of the drugs in various concentrations onto solid $\mathrm{YM}$ agar seeded with $10^{6}-10^{7}$ spores. The plates were incubated at $30^{\circ} \mathrm{C}$ for $2-3 \mathrm{~d}$ and the diameters of the inhibition zones were measured.

Genetic mapping. Genetic mapping of the ebr mutations by conjugation was based primarily on the procedures of Hopwood et al. (1985). Spores of an $e b r$ mutant were mixed with spores of S. lividans 3078 (about $10^{7}$ of each), and grown on R2 medium until sporulation occurred ( $7-10 \mathrm{~d})$. The spores were plated on $\mathrm{MM}$ containing leucine, proline and spectinomycin to select for $b i s^{+} s p c$ recombinants. The genotypes of the recombinants were further analysed by replica plating on diagnostic media containing various combinations of supplements.

Uptake and efflux of EB. The fluorescence technique used by Jones \& Midgley (1985) and Midgley (1986) was adapted to measure the uptake and efflux of EB. Mycelium was collected from an early exponential culture in TSB medium, washed three times and resuspended in ice-cold MM (without glucose; filtered through a $0 \cdot 2 \mu \mathrm{m}$ polyestersulfone membrane) to an $\mathrm{OD}_{600}$ of $0 \cdot 3$, and kept on ice.

For the measurement of EB accumulation, $2 \mathrm{ml}$ of the suspended mycelium was transferred to a cuvette in a Hitachi F-4010 fluorescence spectrophotometer and stirred with a magnetic stir bar. Glucose $(1 \mathrm{M})$ was added to a final concentration of $10 \mathrm{mM}$. To test the energy dependence of the accumulation and efflux, the oxidative phosphorylation uncoupler CCCP $(20 \mathrm{mM})$ was added in parallel with glucose to a final concentration of $40 \mu \mathrm{M}$. Uptake was initiated when EB $(2.5 \mathrm{mM})$ was added to a final concentration of $5 \mu \mathrm{M}$, and fluorescence was measured at $596 \mathrm{~nm}$ using a $530 \mathrm{~nm}$ excitation light. Mycelial aggregates formed during the measurement were intermittently broken up by repeated pipetting through a $1 \mathrm{ml}$ pipette.

For efflux measurements, the washed mycelium $\left(\mathrm{OD}_{600} 0.3\right)$ was pre-incubated in $\mathrm{MM}$ containing $5 \mu \mathrm{M} \mathrm{EB}, 40 \mu \mathrm{M} \mathrm{CCCP}$, and $10 \mathrm{mM}$ glucose at $30^{\circ} \mathrm{C}$ for $1 \mathrm{~h}$, washed three times in ice-cold MM (without glucose), and resuspended in $0 \cdot 1$ vol. MM (without glucose) immediately before assay. The cuvette was first filled with $1.8 \mathrm{ml} \mathrm{MM}$ (containing glucose and/or CCCP as necessary), and the measurement of fluorescence was started when $0.2 \mathrm{ml}$ of EB-preloaded mycelium was added. When protoplasts were used for the assays, they were prepared from fresh cultures, and all the media contained $0 \cdot 3 \mathrm{M}$ sucrose.

\section{RESULTS}

\section{Low resistance of S. lividans TK64 to EB}

S. lividans TK64 exhibited various degrees of resistance to low concentrations (below $8 \mu \mathrm{M}$ ) of $\mathrm{EB}$ as a function of the medium composition (Fig. 1). The survival curves displayed a shoulder up to an EB concentration of $2 \mu \mathrm{M}$ in YM medium, and $6 \mu \mathrm{M}$ in the richer $\mathrm{CM}$ medium. Further supplementation of glucose $(1 \%)$ to $\mathrm{YM}$ slightly increased the survival fraction of TK64, while removal of glucose from CM reduced the survival fraction. These results indicated that richer nutrients increased the resistance of TK64 to EB.

To test whether EB resistance was inducible, TK64 was grown for a $0 \cdot 5-8 \mathrm{~h}$ on $\mathrm{YM}$ containing a sublethal

Table 1. S. lividans strains used in this study

\begin{tabular}{|c|c|c|}
\hline Designation & Genotype/characteristics & Source/reference \\
\hline 1326 & Wild-type & Hopwood et al. (1983) \\
\hline 3078 & bis-2 leu-2 spc-1 pI J303 SLP2- SLP3- & Hopwood et al. (1983) \\
\hline TK64 & pro-2 str-6 SLP2- SLP3- & Hopwood et al. (1983) \\
\hline YJ11 & pro-2 str-6 ebr-11 SLP2- SLP3- & This study \\
\hline YJ18 & pro-2 str-6 ebr-18 SLP2- SLP3- & This study \\
\hline YJ51 & pro-2 str-6 ebr-51 SLP2- SLP3- & This study \\
\hline YJ52 & pro-2 str-6 ebr-52 SLP2- SLP3- & This study \\
\hline YJ57 & pro-2 str-6 ebr-57 SLP2- SLP3- & This study \\
\hline
\end{tabular}




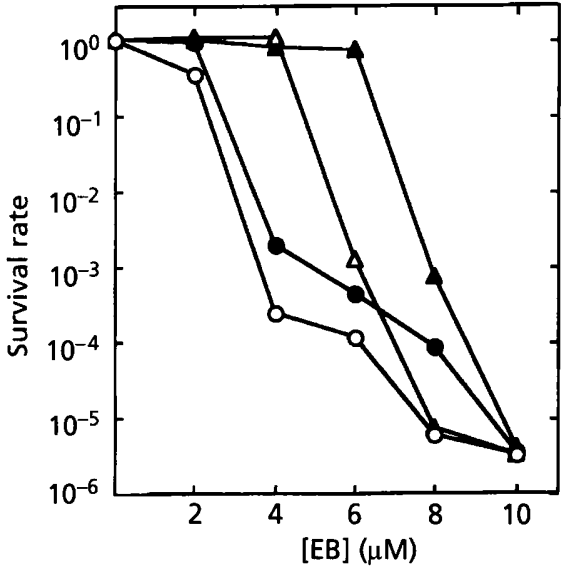

Fig. 1. Sensitivity of S. lividans TK64 to low concentrations of EB. O, YM agar;, YM agar plus $1 \%$ glucose; $\Delta, C M$ agar; $\triangle$, $\mathrm{CM}$ minus glucose.

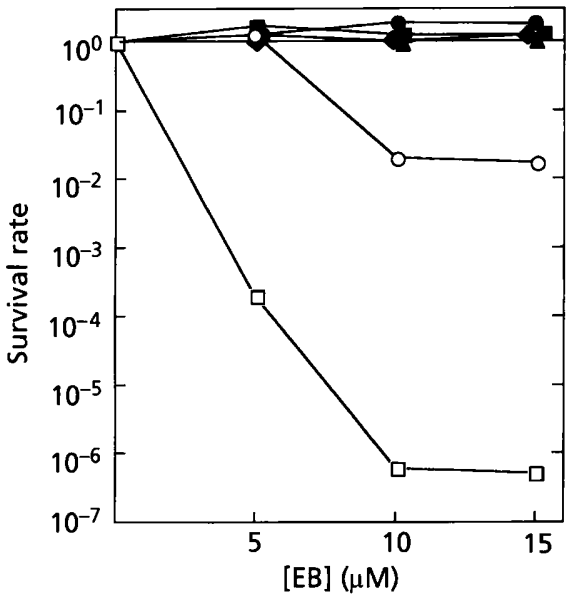

Fig. 2. Sensitivity of S. lividans TK64 and its ebr derivatives to $E B$. The survival fractions (on YM agar) were calculated as the ratios of the numbers of colony-forming units in the presence and absence of EB. $\square$, TK64; $\bullet, Y J 11 ; O, Y J 18 ; \square, Y J 51 ; 0$, YJ52; $\Delta$, YJ57.

concentration $(1 \mu \mathrm{M})$ of EB before challenge with a lethal concentration of EB. No increase in the survival fraction was observed for EB concentrations up to $10 \mu \mathrm{M}$.

\section{Isolation of EB-resistant (ebr) mutants}

The colony-forming ability of S. lividans TK64 spores on YM containing 10 and $15 \mu \mathrm{M}$ EB was reduced by about 6 orders of magnitude (Fig. 2). Five spontaneous resistant mutants were picked from amongst the surviving colonies, and designated Y J11, Y J18, Y J51, Y J52 and Y J57 (carrying mutations ebr-11,ebr-18,ebr-51,ebr-52 and ebr57 , respectively). Four of the mutants exhibited essentially complete resistance to EB up to $15 \mu \mathrm{M}$; the fifth mutant, YJ18, was partially sensitive to $E B$ at concentrations above $5 \mu \mathrm{M}$ (Fig. 2). The four totally resistant mutants
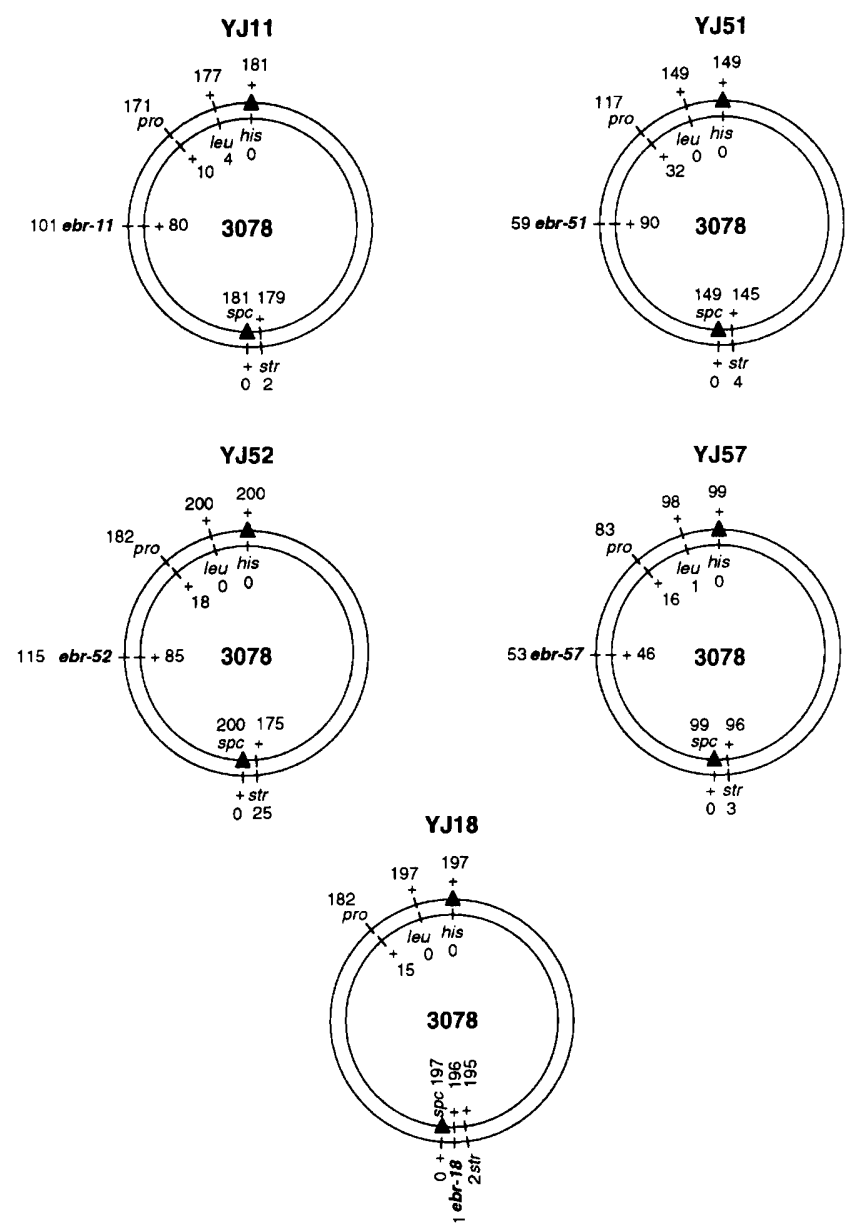

Fig. 3. Genetic mapping of the ebr mutations in S. lividans. The circles represent the genetic maps of the two mating strains (inside, 3078; outside, the ebr mutant). The triangles indicate the selected alleles ( his $^{+}$and spc-1).

(YJ11, YJ51, YJ52 and YJ57) were grouped as class I, and the partially resistant mutant, YJ18, as class II.

The morphology, pigmentation and growth characteristics of these mutants were indistinguishable from those of TK64. Total DNA from the ebr mutants, analysed by conventional restriction digestion and electrophoresis, showed no detectable amplifications. Digestion of the chromosomal DNA with the rare-cutting enzyme AseI and separation by pulsed-field gel electrophoresis gave rise to the same 16 chromosomal fragments (Leblond $e t$ al., 1993; Lin et al., 1993) as in TK64. These results indicated that no gross genomic rearrangements had occurred in these ebr mutants, despite their prior exposure to the rearrangement-inducing EB.

\section{Genetic mapping of the ebr mutations}

The $e b r$ mutations were mapped by conjugation. Each of the $e b r$ mutants (pro-2 str-6) was mated with S. lividans 3078 (bis-2 leu-2 spc-1) containing the conjugative plasmid pIJ303 (Hopwood et al., 1983). $\mathrm{His}^{+}$and spectinomycinresistant $(s p c)$ recombinants were isolated and analysed for 

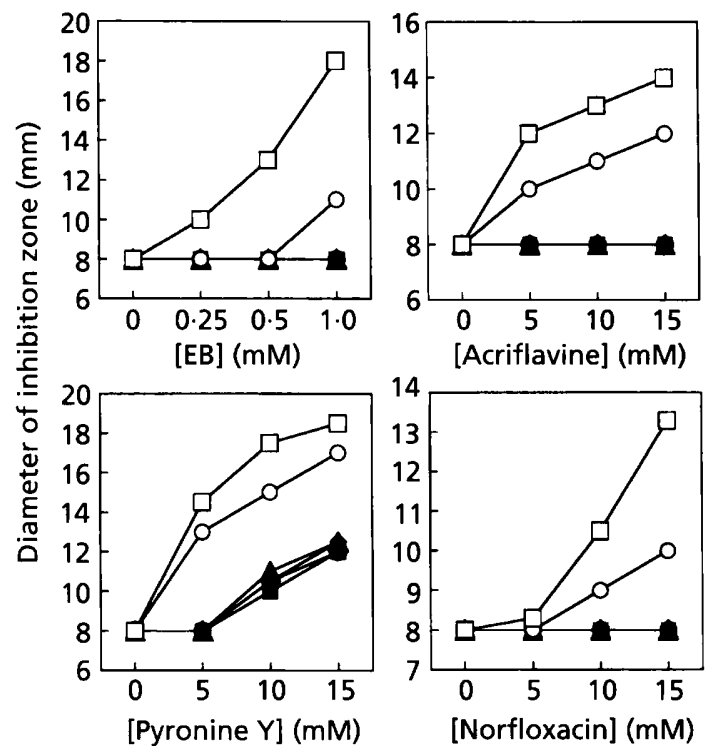
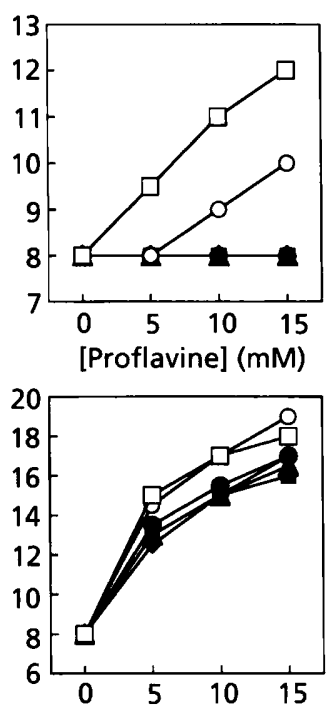

[Methyl green] (mM)
Fig. 4. Sensitivities of the ebr mutants to other drugs. The filter disk assay was used to measure the mean diameter of the inhibition zones exerted by drugs applied to the filter disks. The diameter of the filters was $8 \mathrm{~mm}$. $\square$, TK64; $\diamond, Y J 11 ; O, Y J 18 ; \square$. YJ51; O, YJ52; $\Delta$, YJ57. The results are summarized in Table 2. the segregation of the unselected markers. The frequency of $b i s^{+} s p c$ recombinants in each mating was $2-9 \times 10^{-3}$. From the allele ratios of the markers there were two possible locations for each of the ebr mutations: between pro-2 and $s p c-1$ or between bis-2 and str- 6 for the class I mutations; and on the left or right of $s p c-1$ for the class II mutation (Fig. 3). The choice was made between each pair of locations by analysing the linkage between each ebr mutation and its neighbouring markers by minimizing the numbers of crossovers required for the recombinant genotypes (Hopwood et al., 1985). Class I mutations were placed in the 9 o'clock region, and the class II mutation between str- 6 and $s p c-1$ near 6 o'clock.

\section{Cross-resistance of the ebr mutants to other DNA- intercalating agents}

EB resistance mutations generally affect susceptibility of the mutants to other drugs. The sensitivities of these mutants to three other DNA intercalators, acriflavine, proflavine and acridine orange, were similar to their sensitivity to EB: the class I mutants were completely resistant, and the class II mutant was partially resistant (Fig. 4; Table 2). The class I mutants were also more resistant to pyronine $\mathrm{Y}$ than the class II mutant. The class I mutants showed a slightly lower sensitivity to crystal violet and methyl green than TK64, while Y J18 was only slightly less sensitive to $\mathrm{CV}$ than TK64. The results indicated a biochemical difference in the resistance mechanisms in the two classes of $e b r$ mutants, further justifying the classification.

The ebr mutants were as sensitive as TK64 to rhodamine6G, SDS, CCCP and two quaternary ammonium compounds, cetyltrimethylammonium bromide and benzalkonium chloride. They were also equally resistant as TK64 to two basic dyes, toluidine blue $\mathrm{O}$ and methylene blue. Y J18 was as sensitive as TK64 to the phosphonium ions methyl-triphenylphosphonium bromide and tetraphenylphosphonium bromide, while YJ51 showed re- sistance (Table 2). All ebr mutants exhibited elevated resistance to norfloxacin, a fluoroquinolone; the class I mutants were more resistant than the class II mutant (Fig. 4).

These results showed that the $e b r$ mutations had relatively broad ranges of resistance spectra, like the multidrug resistance systems in other bacteria. TK64 is a derivative of the wild-type strain 1326 obtained through several steps of mutations (Hopwood et al., 1983). The pattern of drug susceptibility of 1326 was identical to that of TK64, except for the streptomycin resistance conferred by a mutation in TK64 (Hopwood et al., 1983).

\section{Efflux studies}

The uptake and efflux of EB by mycelial fragments of Y J18 and Y J51 were compared to those of TK64 using a fluorescence assay (Jones \& Midgley, 1985; Midgley, 1986), in which the presence of intracellular EB was measured by the fluorescence produced by the EB-nucleic acid complexes (Fig. 5, left panels). Both TK64 and Y J18 accumulated EB in minimal medium (containing glucose), approaching a plateau in about $40 \mathrm{~min}$. Addition of CCCP, an uncoupler of oxidative phosphorylation, resulted in an approximately $70 \%$ increase in accumulation, indicating the existence of an energy-dependent efflux system in these two strains. YJ51, on the other hand, accumulated much less EB and approached a plateau in less than $5 \mathrm{~min}$ in the absence of CCCP. It accumulated about three times more EB in the presence of CCCP. This indicated that the putative efflux system in YJ51 was significantly more efficient than those in TK64 and YJ18.

The presence of the efflux system was supported by efflux studies (Fig. 5, right panels), in which the efflux of EB was measured after removal of EB from the medium. TK64 and Y J18 pumped out EB at about the same rate, reaching a basal level in $40 \mathrm{~min}$. In contrast, EB efflux in Y J51 was much faster and completed in less than 10 min. The efflux 
Table 2. Summary of drug resistance patterns of the ebr mutants

See Fig. 4 for representative quantitative results.

\begin{tabular}{|lccc|}
\hline \multirow{2}{*}{ Drug } & \multicolumn{2}{c|}{ Resistance/sensitivity* } \\
\cline { 2 - 4 } & TK64 & Class I & Class II \\
\hline Basic dyes & & & \\
EB & $\mathrm{S}$ & $\mathrm{R}$ & $\mathrm{r}$ \\
Acriflavine & $\mathrm{S}$ & $\mathrm{R}$ & $\mathrm{r}$ \\
Proflavine & $\mathrm{S}$ & $\mathrm{R}$ & $\mathrm{r}$ \\
Pyronine Y & $\mathrm{S}$ & $\mathrm{R}$ & $\mathrm{r}$ \\
Crystal violet & $\mathrm{S}$ & $\mathrm{r}$ & $\mathrm{r}$ \\
Methyl green & $\mathrm{S}$ & $\mathrm{r}$ & $\mathrm{S}$ \\
Toluidine blue O & $\mathrm{R}$ & $\mathrm{R}$ & $\mathrm{R}$ \\
Methylene blue & $\mathrm{R}$ & $\mathrm{R}$ & $\mathrm{R}$ \\
Rhodamine-6G & $\mathrm{S}$ & $\mathrm{S}$ & $\mathrm{S}$ \\
Phosphonium ions & & & \\
Methyltriphenylphosphonium & $\mathrm{S}$ & $\mathrm{R} \dagger$ & $\mathrm{S}$ \\
Tetraphenylphosphonium & $\mathrm{S}$ & $\mathrm{R} \dagger$ & $\mathrm{S}$ \\
Quaternary ammonium compounds & & & \\
Cetyltrimethylammonium bromide & $\mathrm{S}$ & $\mathrm{S}$ & $\mathrm{S}$ \\
Benzalkonium chloride & $\mathrm{S}$ & $\mathrm{S}$ & $\mathrm{S}$ \\
Detergent & & & \\
SDS & $\mathrm{S}$ & $\mathrm{S}$ & $\mathrm{S}$ \\
Uncoupler & & & \\
CCCP & $\mathrm{S}$ & $\mathrm{S}$ & $\mathrm{S}$ \\
Fluoroquinolone & & & \\
Norfloxacin & $\mathrm{S}$ & $\mathrm{R}$ & $\mathrm{r}$ \\
\hline
\end{tabular}

* S, sensitive; $\mathrm{R}$, completely/highly resistant; $\mathrm{r}$, partially/slightly resistant.

†Only Y J51 was tested.

in all three cultures was blocked by CCCP. These results confirmed a more efficient efflux system in YJ51, which was presumed to be the result of the ebr-51 mutation and responsible for the elevated resistance of this strain to EB. Another class I mutant, YJ11, was tested; it exhibited a similar increase in EB efflux and a similar decrease in EB accumulation to those seen in YJ51. Similar results were seen when protoplasts were used for the assays instead of mycelial fragments (data not shown).

\section{DISCUSSION}

Wild-type $S$. lividans (represented by TK64) appears to possess at least one functional EB efflux system, which may be responsible for the limited resistance observed in rich medium $(\mathrm{CM})$. On the other hand, the increased efflux shown in the class I $e b r$ mutants appeared to be sufficient for complete resistance to $15 \mu \mathrm{M} \mathrm{EB}$. This type of resistance, mediated by increased efflux, has been well documented in E. coli and other bacteria (Midgley, 1987). The efflux system in wild-type S. lividans is not inducible by EB, so its biological role is not clear. Removal of

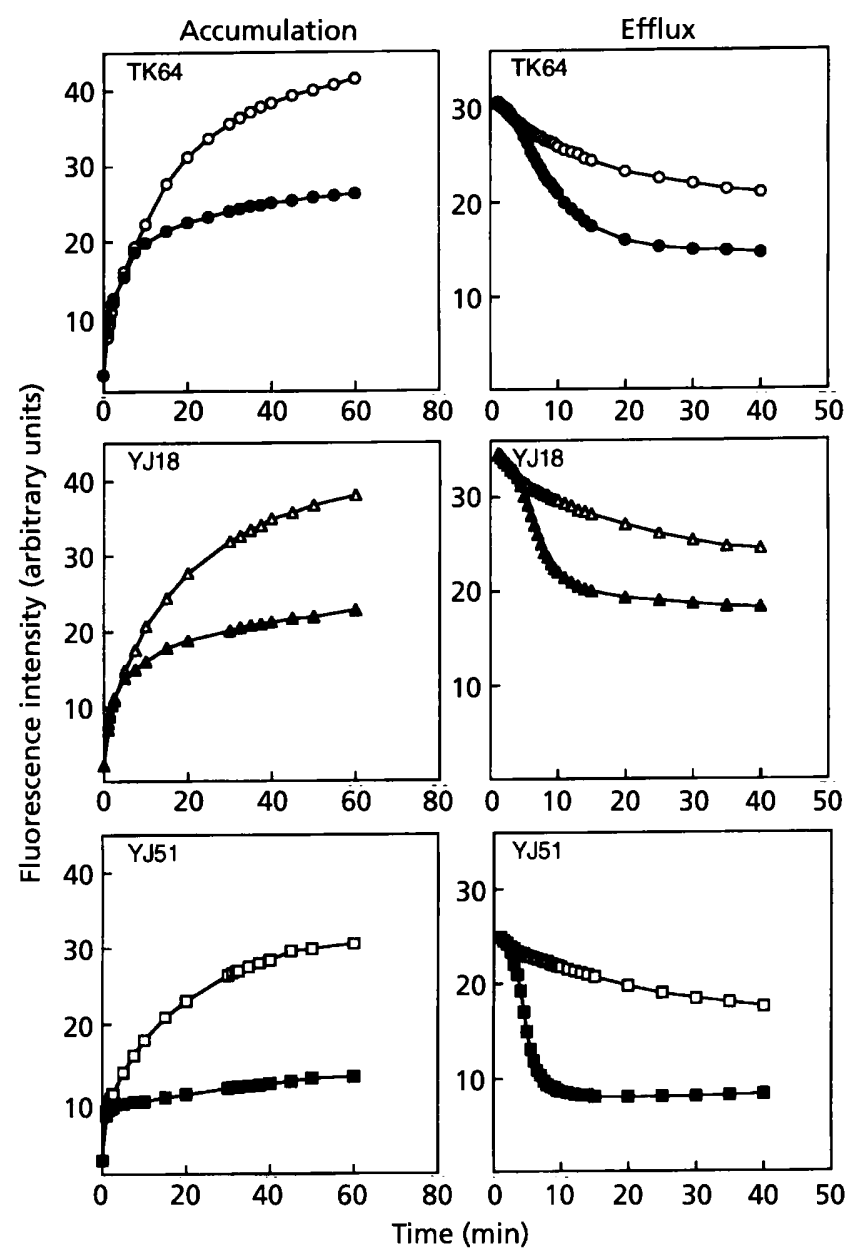

Fig. 5. Accumulation and efflux of EB in the ebr mutants. Left panels: accumulation of intracellular $E B$ by the mycelium of YJ18, YJ51 or TK64 was measured in the presence (open symbols) or absence (filled symbols) of CCCP. Right panels: efflux of EB from the mycelium of YJ18, YJ51 or TK64 was measured in MM containing glucose in the presence (open symbols) or absence (filled symbols) of CCCP.

potentially toxic compounds is one possibility (Ferguson \& Baguley, 1993). The preference for basic dyes as the substrate of the ebr system suggests that it may act to protect the nucleic acids and/or protein targets in the cell.

TK64 growing on rich media exhibited an increased EB resistance (Fig. 1). The difference between $\mathrm{CM}$, which allowed the highest resistance (to $6 \mu \mathrm{M}$ ), and YM, which allowed the least (to $\leqslant 2 \mu \mathrm{M}$ ) may reflect the additional peptone, glucose and $\mathrm{MgCl}_{2}$ in $\mathrm{CM}$. Although the addition of glucose to $\mathrm{YM}$ increased the resistance slightly, CM depleted of glucose still allowed better resistance than YM. Thus, it appeared that the higher resistance in YM was mainly due to the peptone. The putative role of peptone in promoting resistance may be (i) through titration of EB by the peptides in it, (ii) by supporting faster growth and higher cellular DNA content, or (iii) through the induction of one or more efflux systems. 
The two classes of $e b r$ mutations differed in their respective genetic locations, and in the spectra and extent of drug resistance conferred. The class I mutants appeared to acquire increased resistance through elevated energydependent efflux. Although the increased efflux was demonstrated only in YJ51 and YJ11, it is likely to be present also in YJ52 and YJ57.

The class II mutant YJ18 did not exhibit a significant increase in EB efflux. The reproducible sigmoid shape of the EB survival curve of this mutant, with a shoulder extending to $5 \mu \mathrm{M} \mathrm{EB}$, is interesting. Resistance to $\mathrm{EB}$ at concentrations lower than $5 \mu \mathrm{M}$ could not be mediated by an uptake barrier, because YJ18 accumulated EB at this concentration at the same rate as TK64. The resistance may be mediated by a mutation in a cellular target of EB, resulting in loss of affinity. Proteins, in addition to nucleic acids, are targets of EB binding, a notable example being the RecA protein of E. coli (Thresher \& Griffith, 1990). The sensitivity of YJ18 to EB above $5 \mu \mathrm{M}$ might be mediated by a secondary target of lower sensitivity.

Antibiotic-producing cultures of Streptomyces spp. frequently contain efflux systems specific for the antibiotics produced, such as ActII for actinorhodin (Caballero et al., 1991), and Mmr for methylenomycin in Streptomyces coelicolor A3(2) (Neal \& Chater, 1987), OtrB for oxytetracycline in Streptomyces rimosus (Ohnuki et al., 1985), $\operatorname{Drr} A$ and $\operatorname{DrrB}$ for daunorubicin and doxorubicin, respectively, in Streptomyces peucetius (Guilfoile \& Hutchinson, 1991), TlrC and TlrB for tylosin in Streptomyces fradiae (Rosteck et al., 1991), and TcmA for tetracenomycin in Streptomyces glaucescens (Guilfoile \& Hutchinson, 1992). Genes encoding these proteins are closely linked to the production genes. The efflux system(s) identified in this study represent(s) the first broad-spectrum system found in Streptomyces. The ebr mutations of both classes conferred resistance to most of the basic dyes tested, but not to quaternary ammonium ions, SDS or CCCP (Table 2). YJ51 exhibited a slight resistance to phosphonium ions. The broad-spectrum efflux systems characterized in other Gram-positive bacteria exhibited similar substrate spectra. The Bmr transporter of B. subtilis acts on basic dyes, fluoroquinolones, chloramphenicol and puromycin (Neyfakh et al., 1991). In Staphylococcus aureus the QacA and QacC (Smr) systems transport basic dyes and quaternary ammonium compounds (Tennent et al., 1989; Littlejohn et al., 1991); the NorA system transports basic dyes, fluoroquinolones, phosphonium ions, puromycin and chloramphenicol (Yoshida et al., 1990).

The $e b r$ mutants were isolated on medium containing EB at concentrations that may have been high enough to induce chromosomal rearrangements (Gregory \& Huang, 1964; Crameri et al., 1986), so it was possible that the mutations resulted from the mutagenic action of EB. However, several observations argue against this possibility. Firstly, other than the acquired resistance observed in these mutants, there were no phenotypic changes, particularly the loss of chloramphenicol resistance or arginine prototrophy frequently associated with EB- induced deletions. Secondly, no gross DNA arrangements (another EB-sensitive event) were seen in any of the $e b r$ mutants. Thirdly, no new traits were segregated in the recombinant products of the crosses between the $e b r$ mutants and 3078 . Therefore, the ebr mutations appeared to exist before the EB selection.

Chou (1987) found that the rec-46 mutant, JT46, of $S$. lividans that was defective in intraplasmid recombination exhibited slightly higher resistance to EB. However, the $e b r$ mutants were as proficient in plasmid recombination as TK64 (Huang, 1989), indicating the absence of correlation between $\mathrm{EB}$ resistance and intraplasmid recombination.

\section{ACKNOWLEDGEMENTS}

This study was supported by research grants from the National Science Council, ROC (NSC83-0203-B010-067 and NSC842331-B010-054). C.W.C. was a recipient of a research award from the Medical Research and Advancement Foundation in memory of Chi-Shuen Tsou. We thank Professor David A. Hopwood for critical reading of the manuscript and helpful comments.

\section{REFERENCES}

Brenner, S., Barnet, L., Crick, F. H. C. \& Orgel, A. (1961). The theory of mutagenesis. J Mol Biol 3, 121-124.

Caballero, J. L., Malpartida, F. \& Hopwood, D. A. (1991). Transcriptional organization and regulation of an antibiotic export complex in the producing Streptomyces culture. Mol Gen Genet 228, 372-380.

Chou, C. F. (1987). Study on a recombination gene in Streptomyces. $\mathrm{PhD}$ thesis, Yang-Ming Medical College, Taipei, Taiwan.

Crameri, R., Davies, J. E. \& Hütter, R. (1986). Plasmid curing and generation of mutations induced with ethidium bromide in streptomycetes. J Gen Microbiol 132, 819-824.

Crick, F. H. C., Barnet, L., Brenner, S. \& Watts-Tobin, R. J. (1961). General nature of the genetic code for proteins. Nature 192, $1227-1232$.

Dyson, P. \& Schrempf, H. (1987). Genetic instability and DNA amplifications in Streptomyces lividans 66. J Bacteriol 169, 4796-4803.

Ferguson, L. R. \& Baguley, B. C. (1993). Multidrug resistance and mutagenesis. Mutat Res 285, 79-90.

Gregory, K. F. \& Huang, J. C. C. (1964). Tyrosinase inheritance in Streptomyces scabies. II. Induction of tyrosinase deficiency by acridine dyes. J Bacteriol 87, 1287-1294.

Guilfoile, P. G. \& Hutchinson, C. R. (1991). A bacterial analog of the $m d r$ gene of mammalian tumor cells is present in Streptomyces peucetius, the producer of daunorubicin and doxorubicin. Proc Natl Acad Sci US A 88, 8553-8557.

Guilfoile, P. G. \& Hutchinson, C. R. (1992). Sequence and transcriptional analysis of the Streptomyces glaucescens tcm $\mathrm{AR}$ tetracenomycin $C$ resistance and repressor gene loci. J Bacteriol 174, 3651-3658.

Hardin, C. C., Walker, G. T. \& Tinoco, I., Jr (1988). Binding of ethidium ion to left-handed Z-RNA induces a cooperative transition to right-handed RNA at the intercalating site. Biochemistry 27, 4178-4184.

Hintermann, G., Crameri, R., Vögtli, M. \& Hütter, R. (1984). Streptomycin sensitivity by Streptomyces glauscens is due to deletions comprising the structural gene encoding for a specific phosphotransferase. Mol Gen Genet 196, 513-520. 
Hopwood, D. A., Kieser, T., Wright, H. M. \& Bibb, M. J. (1983). Plasmids, recombination, and chromosomal mapping in Streptomyces lividans 66. J Gen Microbiol 129, 2257-2269.

Hopwood, D. A., Bibb, M. J., Chater, K. F., Kieser, T., Bruton, C. J., Kieser, H. M., Lydiate, D. J., Smith, C. P., Ward, J. M. \& Schrempf, H. (1985). Genetic Manipulation of Streptomyces: a Laboratory Manual. Norwich, UK: John Innes Foundation.

Huang, Y.-J. (1989). Isolation of ethidium bromide resistant mutants of Streptomyces lividans, and mapping and cloning of the resistance genes. MS thesis, Yang-Ming Medical College, Taipei, Taiwan.

Jones, I. G. \& Midgley, M. (1985). Expression of a plasmid borne ethidium resistance determinant from Staphylococcus in Escherichia coli: evidence for an efflux. FEMS Microbiol Lett 28, 355-358.

Leblond, P., Redenbach, M. \& Cullum, J. (1993). Physical map of the Streptomyces lividans 66 and comparison with that of the related strain Streptomyces coelicolor A3(2). J Bacteriol 175, 3422-3429.

Lewis, K. (1994). Multidrug resistance pumps in bacteria : variations on a theme. Trends Biocbem Sci 19, 119-123.

Lin, Y.-S., Kieser, H. M., Hopwood, D. A. \& Chen, C. W. (1993). The chromosomal DNA of Streptomyces lividans 66 is linear. Mol Microbiol 10, 923-933.

Littlejohn, T. G., DiBerardino, D., Messerotti, L. J., Spiers, S. J. \& Skurray, R. A. (1991). Structure and evolution of a family of genes encoding antiseptic and disinfectant resistance in Stapbylococcus aureus. Gene 101, 59-66.

Ma, D., Cook, D. N., Hearst, J. E. \& Nikaido, H. (1994). Efflux pumps and drug resistance in Gram-negative bacteria. Trends Microbiol 2, 489-493.

Midgley, M. (1986). The phosphonium ion efflux system of Escbericbia coli: relationship to the ethidium efflux system and energetic studies. J Gen Microbiol 132, 3187-3193.

Midgley, M. (1987). An efflux system for cationic dyes and related compounds in Escherichia coli. Microbiol Sci 4, 125-127.

Neal, R. J. \& Chater, K. F. (1987). Nucleotide sequence analysis reveals similarities between proteins determining methylenomycin A resistance in Streptomyces and tetracycline resistance in eubacteria. Gene 58, 229-241.

Neyfakh, A. A., Bidnenko, V. E. \& Chen, L. B. (1991). Effluxmediated multidrug resistance in Bacillus subtilis: similarities and dissimilarities with the mammalian system. Proc Natl Acad Sci USA 88, 4781-4785.

Nikaido, H. (1994). Prevention of drug access to bacterial targets: permeability barriers and active efflux. Science 264, 382-388.

Ohnuki, T., Kaith, T., Imanaka, T. \& Aiba, S. (1985). Molecular cloning of tetracycline resistance genes from Streptomyces rimosus in
Streptomyces griseus and characterization of the cloned genes. J Bacteriol 161, 1010-1016.

Redenbach, M., Flett, F., Piendl, W., Glocker, I., Rauland, U., Wafzig, O., Leblond, P. \& Cullum, J. (1993). The Streptomyces lividans 66 chromosome contains a $1 \mathrm{Mb}$ deletogenic region flanked by two amplifiable regions. Mol Gen Genet 241, 255-262.

Rosteck, P. R., Reynolds, P. A. \& Hershberger, C. L. (1991). Homology between proteins controlling Streptomyces fradiae tylosin resistance and ATP-binding transport. Gene 102, 27-32.

Rouch, D. A., Cram, D. S., DiBerardino, D., Littlejohn, T. G. \& Skurray, R. A. (1990). Efflux-mediated antiseptic resistance gene qac $A$ from Staphylococcus aureus: common ancestry with tetracyclineand sugar-transport proteins. Mol Microbiol 4, 2051-2062.

Sasatsu, M., Shima, K., Shibata, Y. \& Kono, M. (1989). Nucleotide sequence of a gene that encodes resistance to ethidium bromide from a transferable plasmid in Staphylococcus aureus. Nucleic Acids Res 17, 10103.

Schrempf, H. (1983). Deletion and amplification of DNA sequences in melanin-negative variants of Streptomyces reticuli. Mol Gen Genet 189, 501-505.

Seto, H. \& Tomasz, A. (1977). Inhibitors of genetic recombination in pneumococci. Proc Natl Acad Sci US A 74, 296-299.

Tanner, N. K. \& Cech, T. R. (1985). Self-catalyzed cyclization of the intervening sequence RNA of Tetrabymena: inhibition by intercalating dyes. Nucleic Acids Res 14, 6649-6659.

Tennent, J. M., Lyon, B. R., Gillespie, M. T., May, J. W. \& Skurray, R. A. (1985). Cloning and expression of Staphylococcus aureus plasmidmediated quaternary ammonium resistance in Escherichia coli. Antimicrob Agents Chemotber 27, 79-83.

Tennent, J. M., Lyon, B. R., Midgley, M., Jones, I. G., Purewal, A. S. \& Skurray, R. A. (1989). Physical and biochemical characterization of the qac $A$ gene encoding antiseptic and disinfectant resistance in Staphylococcus aureus. J Gen Microbiol 135, 1-10.

Thresher, R. J. \& Griffith, J. D. (1990). Intercalators promote the binding of RecA to double-stranded DNA. Proc Natl Acad Sci US A 87, 5056-5060.

Walker, G. T., Stone, M. P. \& Krugh, T. R. (1985). Ethidium binding to left-handed $(\mathrm{Z})$ DNA's results in regions of righthanded DNA at the intercalating site. Biochemistry 24, 7462-7471.

Yoshida, H., Bogaki, M., Nakamura, S., Ubukata, K. \& Konno, M. (1990). Nucleotide sequence and characterization of the Staphylococcus aureus nor $A$ gene, which confers resistance to quinolones. $J$ Bacteriol 172, 6942-6949.

Received 20 October 1995; revised 12 December 1995; accepted 15 December 1995. 